\title{
Evaluation of Coronary Artery Bypass Graft Using Multi-Detector CT Angiography: Comparative Study
}

\author{
REDA A. ALARABAWY, M.D.*; MAHMOUD A. DAWOUD, M.D.*; TAYMOUR M. ABD ALLA, M.D.** and \\ AMR A. MUBARAK, M.D.*
}

The Departments of Radiodiagnosis \& Medical Imaging* and Cardiology**, Faculty of Medicine, Tanta University, Egypt

\begin{abstract}
Background: Recurrence of ischemic symptoms after coronary artery bypass graft surgery requires imaging assessment of bypass graft lumen to rule-out graft dysfunction.

Aim of Study: Is to evaluate the role of 320-row multidetector CT as a non-invasive imaging modality for assessment of coronary artery bypass grafts.

Results: Twenty one patients were referred to assess the patency of coronary artery bypass grafts. All patients were subjected to history taking, clinical examination as well as CT angiography of coronary arteries and coronary artery bypass grafts using 320-row multidetector CT scanner after heart rate control and injection of non-ionic contrast material. Total number of 63 interpretable coronary artery bypass grafts were included in this study. On evaluating 23 arterial grafts, all of LIMA grafts were patent without stenosis except for LIMA to D2 graft which shows subtotal distal anastomotic site occlusion. The only RIMA graft in this study was occluded whereas radial artery graft to OM was patent. On evaluating 40 venous grafts, $11(27.5 \%)$ grafts were considered patent, $3(7.5 \%)$ grafts showed significant ( >!50) stenosis and 26 $(65 \%)$ grafts were occluded.
\end{abstract}

Conclusion: Multidetector CT is considered convenient and reliable non-invasive imaging modality for assessment of suspected graft dysfunction.

Key Words: Multidetector CT - Evaluation - Coronary artery bypass grafts.

\section{Introduction}

AMONG the available treatment options, Coronary Artery Bypass Graft (CABG) surgery still remains the standard of care in the treatment of advanced coronary artery disease [1].

Recurrent chest pain after myocardial revascularization is a common clinical presentation. The long-term clinical outcome after surgery is depend-

Correspondence to: Dr. Reda A. Alarabawy, E-Mail: reda.alarabawy@yahoo.com ent on the patency of the bypass grafts and the progression of native coronary artery disease [2]

Conventionally, invasive coronary angiography has been used to assess bypass graft status and to evaluate native coronary arteries. However, it is an invasive procedure and associated with certain risks and complications, such as arrhythmia, graft dissection, myocardial infarction, and embolic events [3].

MDCT has assumed an integral role in imaging of coronary artery bypass grafts while allowing investigation of other post-operative complications. In addition, the expanded capabilities of MDCT may provide valuable information in pre-operative planning for repeating CABG surgery [4].

Our study aimed to evaluate the role of 320row multidetector CT as a non-invasive imaging modality for assessment of coronary artery bypass grafts.

\section{Patients and Methods}

This prospective study was performed at the period from August 2017 to August 2019 including 21 patients, underwent CBAG surgery.

\footnotetext{
Abbreviations:

MDCT : Multidetector Computed Tomography.

CABG : Coronary Artery Bypass Graft.

BMI : Body Mass Index.

LIMA : Left Internal Mammary Artery.

RIMA : Right Internal Mammary Artery.

OM : Obtuse Marginal.

CTA : Computed Tomography Angiograpgy.

LAD : Left Anterior Descending.

LCx : Left Circumflex.

PDA : Posterior Descending Artery.

SVG : Saphenous Vein Graft.

RCA : Right Coronary Artery.
} 
Multidetector CT angiography of coronary arteries was done to all patients referred to Diagnostic Radiology and Medical Imaging Department at our institution from Cardiology Department and private clinics.

Inclusion criteria for this study:

- Recurrent chest pain after previous coronary artery bypass graft surgery.

- Patient underwent previous CABG surgery and had positive results for myocardial ischemia during regular follow-up on doing non-invasive tests like myocardial perfusion scintigraphy, stress echocardiography or stress ECG.

\section{Exclusion criteria for this study:}

- Patient cannot withstand the duration or technique of CT examination.

- Patient with allergy to IV contrast material or had impaired renal function.

The selected 21 patients were subjected to radiological examination including MDCT angiography of coronary arteries, out of them 15 patients underwent invasive coronary angiography after MDCT later on by the referring cardiologist as a gold standard for comparison of CT results.

\section{MDCT angiography of coronary arteries:}

\section{Patient preparation:}

-Instructions: Patients were given instruction to follow before the scheduled CT appointment which included:

- Fasting 4-6 hours before scan ... but encourage water intake.

- Avoid caffeine products, smoking \& exercise 12 hours before scan.

- Stop taking drugs used for impotence or pulmonary hypertension 48 hours before scan.

- Heart rate control: Those with heart rate below $65 \mathrm{bpm}$ (6 patients) were proceeded directly to the scan. Those with resting heart rate ranging from $65-70 \mathrm{bpm}$ (8 patients) and those with heart rate above $70 \mathrm{bpm}$ (5 patients) were given $50 \&$ $100 \mathrm{mg}$ oral Metoprolol respectively one hour prior to scan provided that there was no contraindication to its use, in order to achieve a resting heart rate below $60 \mathrm{bpm}$. Two patients with contraindication to B-blockers use were given $5 \mathrm{mg}$ oral Ivabradine one hour prior to scan.

- IV access: After heart rate control, $18 \mathrm{~g}$ cannula was inserted into right antecubital vein for all patients, left upper limb cannulation was avoided to avoid streak artifact from dense contrast material within left innominate which may interfere with optimal visualization of left internal mammary artery origin, if it was used as a graft.

- At scanner room: Patients lied supine with their feet entering the gantry first and they were positioned to ensure the heart lies in center of the gantry. Four ECG electrodes were placed on their chest after proper skin preparation and were connected by four leads to the CT trigger monitor. Patients were given instructions about how to perform breath holding during the scan and finally received $5 \mathrm{mg}$ sublingual isosorbide dinitrate immediately prior to scan if no contraindication to its use.

Contrast medium injection: Non-ionic contrast media (Ultravist $370 \mathrm{mgI} / \mathrm{ml}$ ) was injected through the peripherally inserted IV cannula using dualhead powered automatic injector followed by $50 \mathrm{cc}$ saline. The flow rate ranged from 4 to $6 \mathrm{ml} / \mathrm{sec}$ while the amount of contrast material ranged from $65 \mathrm{ml}$ to $90 \mathrm{ml}$. Automatic bolus tracking method was used to detect contrast media arrival at descending aorta; premonitoring slice $(100 \mathrm{~mA}, 120$ $\mathrm{kV}$ ) was obtained at main pulmonary artery level and a rounded ROI was placed at descending aorta with trigger threshold being set to $180 \mathrm{HU}$.

Scan protocol: All patients were scanned using 320-row MDCT scanner (Aquillion One, Toshiba Medical Systems, Otawara, Japan) installed at our University Hospital. Initial scanogram was obtained first to plan the scanning range of coronary CTA which extended from just above the clavicles down to $1 \mathrm{~cm}$ below the apex of the heart. Acquisition parameters: $0.35 \mathrm{sec}$ gantry rotation time, variable $\mathrm{mA}$ selected automatically by the scanner according to patient body habitus (range: $300-580 \mathrm{~mA}$ ), variable $\mathrm{kV}$ according to $\mathrm{BMI} ; 100 \mathrm{kV}$ for $\mathrm{BMI}$ below $25 \mathrm{Kg} / \mathrm{m}^{2}, 120 \mathrm{kV}$ for BMI $\left(25-32 \mathrm{Kg} / \mathrm{m}^{2}\right)$ and $135 \mathrm{kV}$ for higher BMI. A breathing exercise was performed to measures the heart rate during breath holding period of 10 seconds after which the scanner automatically adjusted the exposure window settings for optimal temporal resolution. Prospective ECG-triggered helical acquisition was performed routinely for patients with heart rate below $60 \mathrm{bpm}$ exposing only $70-80 \%$ of R-R interval. For those with higher heart rate, acquisition was done by widening the exposure window to include $30-80 \%$ of R-R interval. During the rest of R-R interval, $\mathrm{X}$-ray was turned off completely by the scanner to reduce radiation exposure. 


\section{Image reconstruction:}

Images were reconstructed with a slice thickness of $0.5 \mathrm{~mm}$ with $0.3 \mathrm{~mm}$ interval using soft reconstruction kernel (FC03) at $75 \%$ of R-R interval and also at the best diastolic phase. The best systolic phase was reconstructed also if the exposure window included $30-80 \%$ of R-R interval.

\section{Post processing:}

The reconstructed images were transferred to a workstation (Vitrea FX, Vital Images, USA) to review axial images and also to obtain multi-planar reformatted images at sagittal and coronal planes. Also maximum intensity projection images, 3D volume rendered images, semitransparent 3D volume rendered images and curved planar reformations were obtained for detailed assessment of coronary artery bypass grafts and lesion characterization if present.

\section{Image analysis:}

- Assessment of image quality: According to quality of CT images, studies were classified into: Good quality; no motion artifacts, acceptable quality; mild motion artifacts with still diagnostic scan $\&$ poor quality; non-interpretable graft because of significant motion artifacts.

- Assessment of bypass grafts: The obtained MDCT datasets were analyzed with awareness of the previous surgical procedure, number, and location of bypass grafts. The 3D volume rendered images were useful for global assessment of the location and types of grafts used while axial and curved planar reformatted images were useful for graft luminal assessment.

Each bypass graft was divided into three segments: Body of the graft, proximal, and distal anastomotic sites. The grafts were assessed for the presence of stenosis or occlusion. Patency of the graft was demonstrated if its lumen showed uniform contrast enhancement. The grafts which were considered patent were evaluated for the presence of stenosis afterwards. Significant stenosis was defined as a lumen reduction of $50-99 \%$, whereas lumen reduction of $<50 \%$ was considered as mild insignificant stenosis. Stenosis was classified also on the basis of its location in the body of the graft or at the anastomotic sites (proximal or distal). Segmental or total graft occlusion was diagnosed when a graft segment or the whole graft was not opacified with contrast.

- Assessment of native coronary arteries: The coronary segments distal to graft anastomotic sites and non-grafted coronary arteries were evaluated to detect significant stenosis $(>50 \%)$ or occlusion that may contribute to patient's new ischemic symptoms.

- Detection of other CABG surgery complications: The images were also evaluated to detect any other surgical complications that may contribute to patient's symptoms like pleural effusion, pericardial effusion, sternal infection and so on.

\section{Results}

Among the 21 patients who were referred for coronary artery bypass graft assessment, 14 (67\%) were males while $7(33 \%)$ patients were females. Their age ranged from 54 to 78 years with a mean of $65.43 \pm 6.94$ years.

As regards patient's weight and height measurements; their weight ranged from 64 to $98 \mathrm{~kg}$ with a mean of $82.10 \mathrm{~kg}$ whereas their height ranged from 1.63 to $1.73 \mathrm{~m}$ with a mean of $1.68 \mathrm{~m}$. The Body Mass Index (BMI) of those selected patients ranged from 21.63 to 34.06 with a mean of 28.89 (Table 1).

Table (1): Body measurements in the studied groups.

\begin{tabular}{lccl}
\hline & Weight $(\mathrm{kg})$ & Height $(\mathrm{cm})$ & BMI \\
\hline Mean & 82.10 & 1.68 & 28.89 \\
Std. deviation & 13.57 & 0.03 & 4.47 \\
Minimum & 64 & 1.63 & 21.63 \\
Maximum & 98 & 1.73 & 34.06 \\
Std. error & 2.96 & 0.01 & 0.97 \\
\hline
\end{tabular}

Different risk factors for coronary artery disease were present in studied patients including diabetes mellitus, hypertension, dyslipidemia and smoking. Diabetes mellitus was found in $3(14.29 \%)$ cases, hypertension was found in $10(47.62 \%)$ cases, dyslipidemia in $14(66.67 \%)$ cases and smoking was present in $7(33.33 \%)$ cases (Table 2).

Table (2): Frequency of risk factors in both groups.

\begin{tabular}{|c|c|c|c|c|c|c|c|c|}
\hline & \multicolumn{2}{|c|}{ DM } & \multicolumn{2}{|c|}{ HTN } & \multicolumn{2}{|c|}{ Dyslipidemia } & \multicolumn{2}{|c|}{ Smoking } \\
\hline & $\mathrm{N}$ & $\%$ & $\mathrm{~N}$ & $\%$ & $\mathrm{~N}$ & $\%$ & $\mathrm{~N}$ & $\%$ \\
\hline & 3 & 14.29 & 10 & 47.62 & 14 & 66.67 & & 33.33 \\
\hline$p$-value & \multicolumn{2}{|c|}{0.469} & \multicolumn{2}{|c|}{0.133} & \multicolumn{2}{|c|}{0.075} & \multicolumn{2}{|c|}{0.366} \\
\hline
\end{tabular}

DM : Diabetes Mellitus.

HTN: Hypertension.

Among the studied 21 patients, 15 (72\%) patients required heart rate control prior to scan. Their baseline heart rate ranged from 70 to 95 $\mathrm{bpm}$. After giving heart rate control medications, their heart rate at time of scan ranged from 56 to $75 \mathrm{bpm}$. Patients with heart rate below 60bpm, scanning window was set to include only $70-80 \%$ 
of R-R interval to minimize radiation exposure while in those with heart rate above $60 \mathrm{bpm}$, the scanning window was widened to include $30-80 \%$ of R-R interval to include systolic phase images.

MSCT findings in patients underwent $C A B G$ surgery:

A total number of 63 coronary artery bypass grafts were included in this study. They were present at 21 patients whom they were referred for graft patency assessment. Out of them, $23(36.5 \%)$ bypass grafts were arterial whereas $40(63.5 \%)$ bypass grafts were venous. All of those grafts were considered interpretable at MSCT scan images. The mean time between surgery and MSCT examination was 40 months with a range of 4 months to 112 months.

As regards arterial grafts, the Left Internal Mammary Artery (LIMA) was the commonest arterial graft in this study representing $21(91.3 \%)$ grafts out of the 23 arterial grafts included in this study. Out of 21 LIMA grafts, $16(69.7 \%)$ grafts were anastomosed distally to LAD whereas 4 (17.4\%) grafts were anastomosed to D1 and one graft was anastomosed to D2. The remaining 2 arterial grafts were Right Internal Mammary Artery (RIMA) graft anastomosed distally to mid-segment of RCA, and radial artery graft anastomosed proximally to ascending aorta and distally to OM branch (Table 3).

Table (3): Types and number of arterial grafts.

\begin{tabular}{lcc}
\hline Arterial graft type & No. & $\%$ \\
\hline LIMA to LAD & 16 & 69.7 \\
LIMA to D1 & 4 & 17.4 \\
LIMA to D2 & 1 & 4.3 \\
RIMA to RCA & 1 & 4.3 \\
Radial a. to OM & 1 & 4.3 \\
\hline Total & 23 & 100 \\
\hline
\end{tabular}

On evaluating arterial grafts with MSCT, all of LIMA grafts where patent without stenosis except for LIMA to D2 graft which shows subtotal occlusion at distal anastomotic site. The accompanying metallic surgical clips was not interfering with luminal assessment in all patients. LAD, D1 and D2 segments beyond distal anastomotic sites were patent without stenosis apart from 2 cases in whom the distal LAD was not interpretable secondary to severe cardiac motion artifact. The RIMA graft to mid RCA was totally occluded whereas the radial artery graft to OM was patent without stenosis.

As regards venous grafts, they were connected proximally to ascending aorta, whereas distally variable anastomotic sites were present. Among the 40 venous grafts, $10(25 \%)$ grafts were anastomosed distally with Posterior Descending Artery (PDA), 4 (10\%) with LAD, 8 (20\%) grafts with D1 and $18(45 \%)$ grafts with OM.

On evaluating the 40 venous grafts included in this study with MSCT, 11 (27.5\%) grafts were considered patent without stenosis, 3 (7.5\%) grafts showed significant ( $>50)$ stenosis that was present only at graft body and $26(65 \%)$ grafts were occluded either totally or shortly after their origin.

The distribution of MSCT findings among the different venous grafts included in this study was as follows: (Table 4).

Table (4): MSCT findings of venous grafts.

\begin{tabular}{lcccc}
\hline Graft type & Patent & $\begin{array}{c}\text { Significant } \\
\text { stenosis (>_50) }\end{array}$ & Occlusion & Total \\
\hline SVG to PDA & 3 & 0 & 7 & 10 \\
SVG to LAD & 0 & 0 & 4 & 4 \\
SVG to D1 & 3 & 0 & 5 & 8 \\
SVG to OM & 5 & 3 & 10 & 18 \\
\hline Total & 11 & 3 & 26 & 40 \\
\hline
\end{tabular}

- SVG to PDA: 3 (30\%) grafts were patent without stenosis whereas the remaining $7(70 \%)$ grafts was occluded.

- SVG to LAD: All four grafts included in this study were occluded.

- SVG to D1: 3 (37.5\%) grafts were patent without stenosis whereas the remaining $5(62.5 \%)$ grafts were totally occluded.

- SVG to OM: 5 (27.8\%) grafts were patent without stenosis, $3(16.7 \%)$ grafts showed significant (>_50) stenosis while remaining $10(55.5 \%)$ grafts occluded.

The most commonly encountered occluded venous graft was connected to OM branch where 10 grafts were occluded representing $25 \%$ of the examined venous grafts. Out of these 10 occluded grafts, 8 grafts where totally occluded, one graft was occluded $2.0 \mathrm{~cm}$ from its origin and the remaining graft was totally occluded apart from small $1.0 \mathrm{~cm}$ patent nipple just proximal to distal anastomotic site. SVG to OM was also the most common graft that showed significant ( $>50 \%)$ stenosis that was found at the body of 3 grafts representing $7.5 \%$ of the examined venous grafts.

The coronary arterial segments distal to venous graft anastomotic sites were patent without stenosis apart from one case that showed significant stenosis (70\%) just distal to graft anastomotic site. The 
non-grafted coronary arteries were also assessed for possible progression of atherosclerotic disease that may contribute to patient's symptoms. Their assessment revealed no significant disease at 19 cases while two cases showed significant atherosclerotic disease. The first showed totally occluded mid segment of RCA with distal refilling through collaterals. The other case showed severe $(>70 \%)$ distal RCA stenosis.

No other CABG surgery complications were found among the studied 21 patients. All cases were referred during late post-operative period for assessment of graft patency. So, no early postoperative complications were found in this study.

Coronary angiography was done in 15 cases after MSCT for confirmation and comparison. Coronary angiograms revealed the same results as regards all arterial grafts compared to MSCT. On the other hand, out of the studied 24 venous grafts, one graft showed false positive result in the form of insignificant stenosis $(<50 \%)$ by coronary angiography that was overestimated by MSCT.
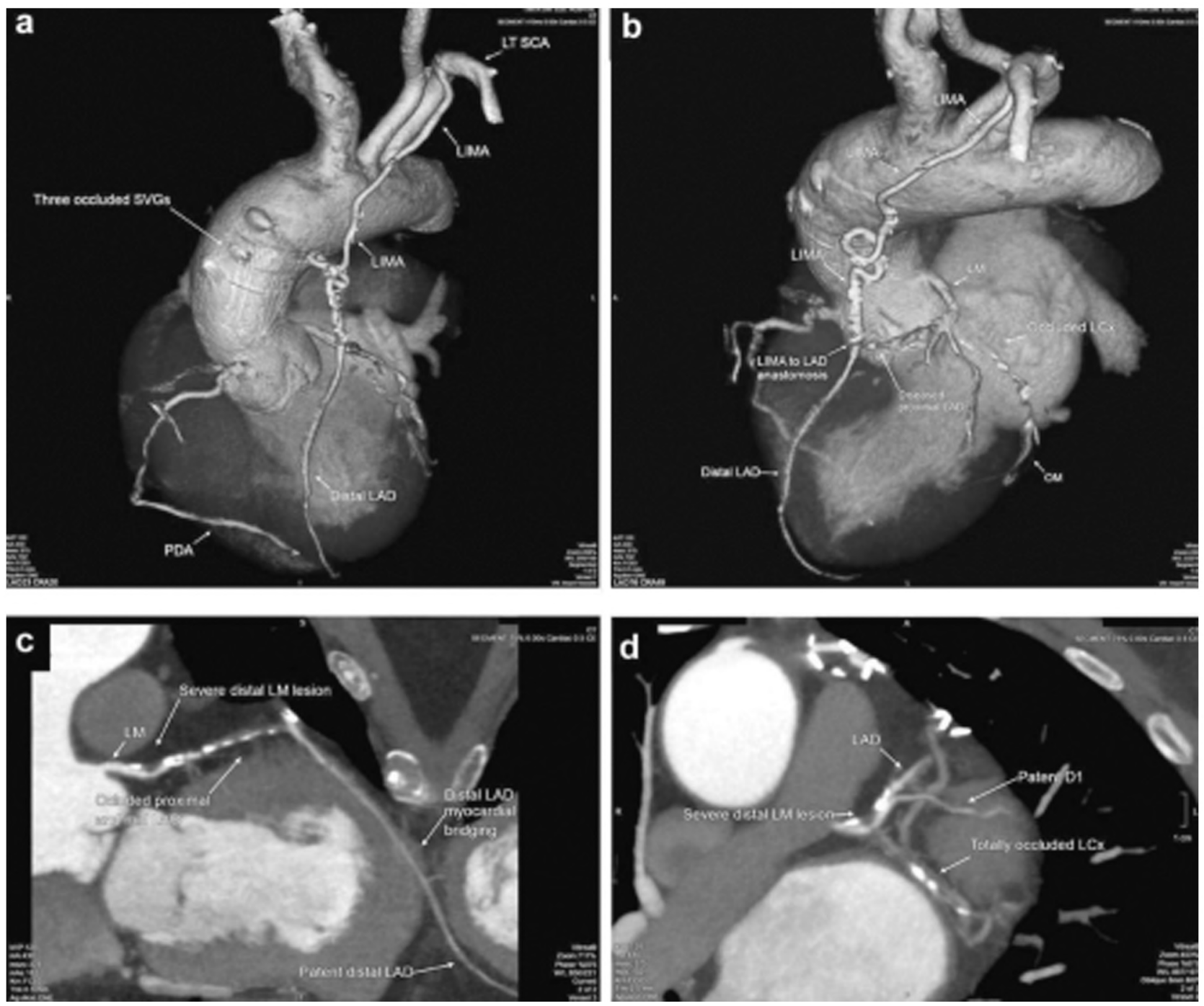

Fig. (1): Three occluded saphenous vein grafts with significant native arteries disease. 3D volume rendered images shows (A \& B) Showing totally occluded three SVGs arising from ascending aorta with patent LIMA to LAD. The curved planar reformatted image (C) Shows severe distal left main coronary artery stenosis and occluded LAD proximal and midsegments with patent distal LAD that shows intramyocardial course. The oblique axial image (D) Shows severe distal left main coronary artery lesion, totally occluded LCx and patent first diagonal branch. 

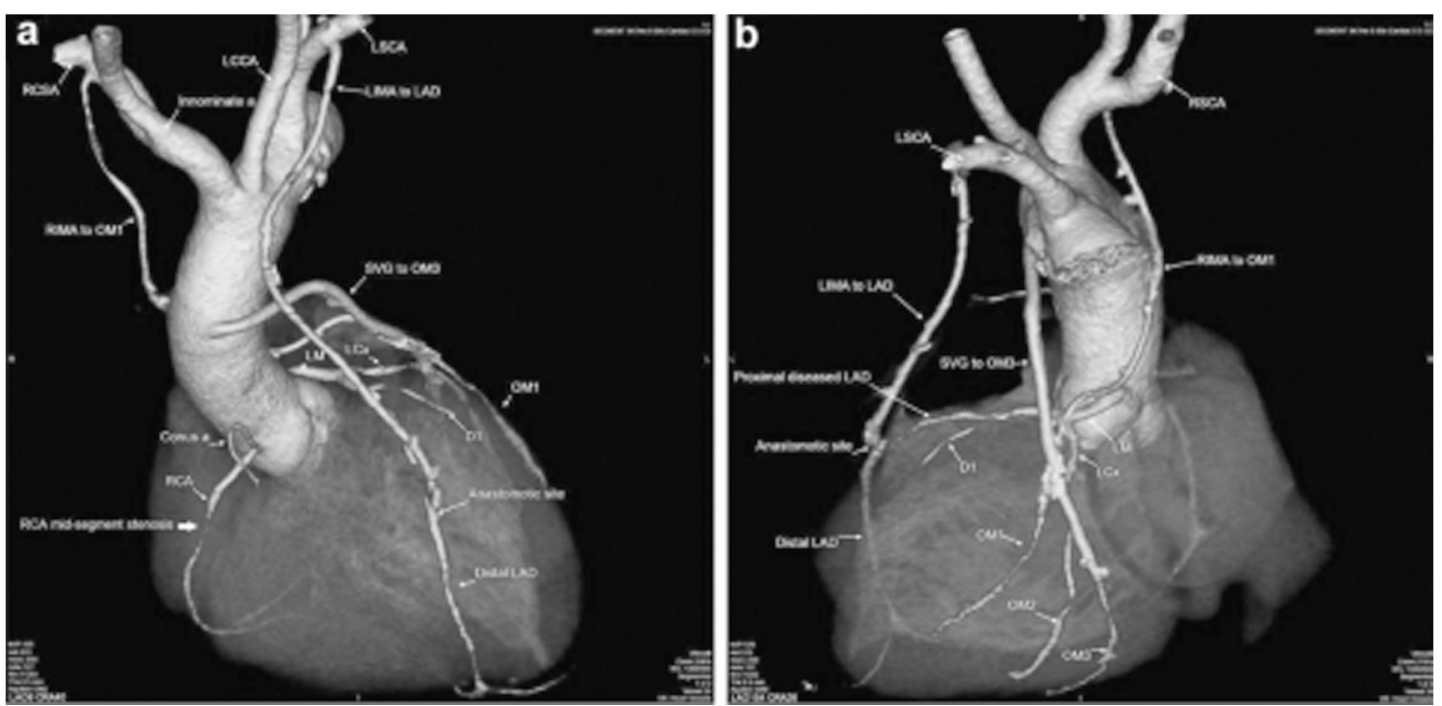

Fig. (2): Patent bypass grafts with significant RCA mid-segment stenosis. 3D volume rendered images (A \& B) Showing patent LIMA to distal LAD, RIMA to OM1 and SVG to OM2 with significant native arteries stenosis seen at RCA midsegment.

LIMA : Left Internal Mammary Artery. RIMA : Right Internal Mammary Artery. LAD : Left Anterior Descending.
SVG : Saphenous Vein Graft. OM : Obtuse Marginal.
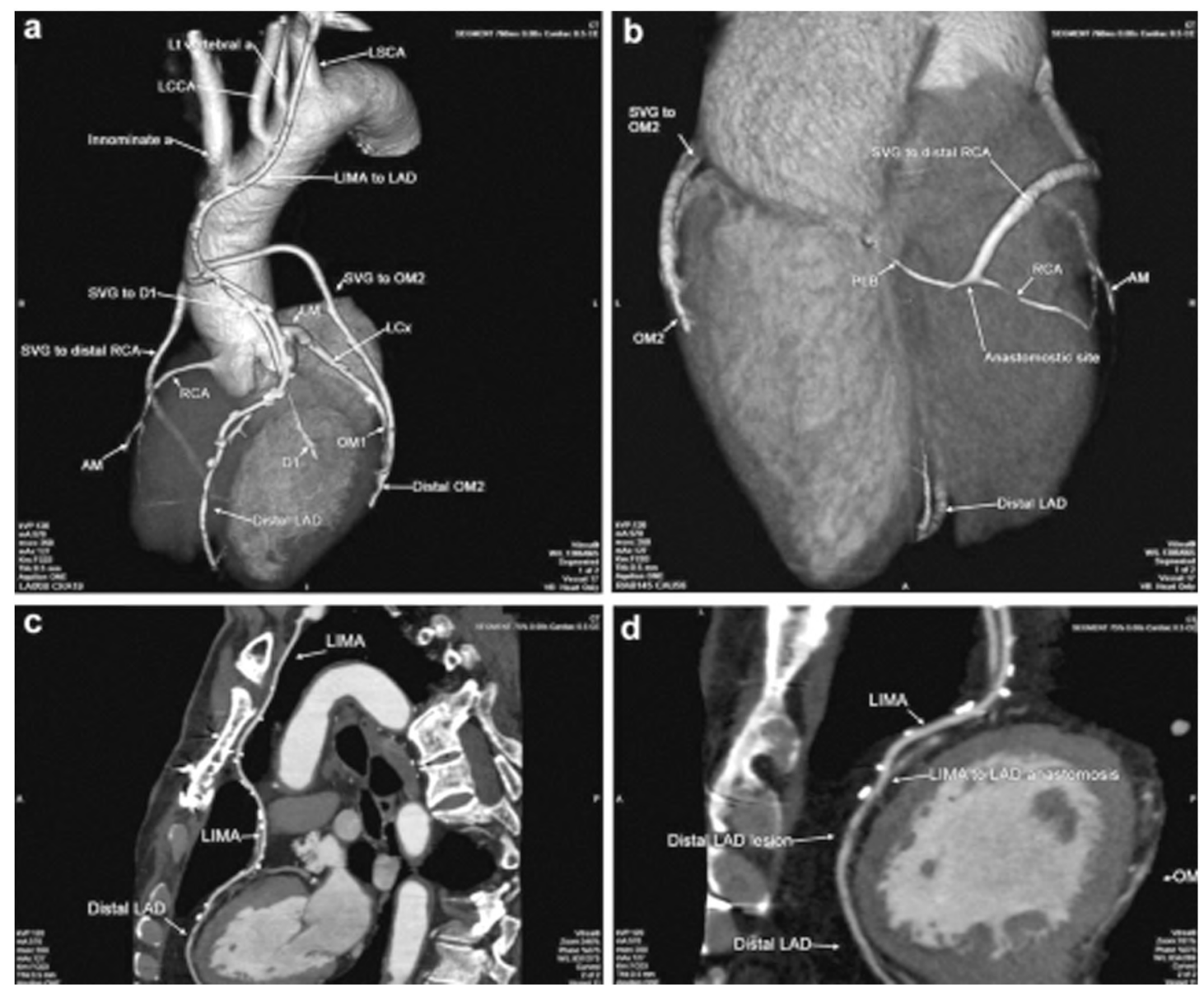

Fig. (3): Significant LAD stenosis beyond LIMA anastomosis. 3D volume rendered images (A \& B) Showing patent LIMA to LAD, SVG to D1, SVG to OM2 and SVG to distal RCA. The curved planar reformatted image (C) Shows patent LIMA to mid LAD without stenosis while the magnified view of distal LAD (D) Shows significant LAD stenosis beyond LIMA anastomosis.

LIMA: Left Internal Mammary Artery. LAD : Left Anterior Descending.
SVG: Saphenous Vein Graft. D : Diagonal.
OM : Obtuse Marginal,

RCA: Right Coronary Artery 


\section{Discussion}

Conventional coronary angiography has been considered the gold standard for evaluation of patients suspected to have coronary artery bypass graft stenosis or occlusion [5].

The use of multi-slice CT is gaining much acceptance for noninvasive cardiac imaging including imaging of coronary artery bypass grafts due to rapid improvement of CT scanners technology

Despite the improvement in CT technology, it is not advisable to scan patients whom their BMI is above $40 \mathrm{kgm}^{2}$ due to significant image noise that interferes with accurate luminal assessment [7]. In this study, the mean BMI for scanned patients was $28.89 \mathrm{kgm}^{-2}$.

Heart rate control the slow heart rate ( $<65 \mathrm{bpm})$ improves the temporal resolution and permits the use of prospective ECG-gating [8]

In study done by Dewey et al. [9] on 30 patients found that radiation exposure reduction was greatest in patients with heart rates $\leqslant 65 \mathrm{bpm}$.

Furthermore, the rapid heart rate can make interpretation of bypass grafts segments close to the heart difficult including distal anastomotic sites secondary to cardiac motion artifact highlighting the importance of heart rate control [9]

MDCT scanners combine a high spatial resolution with the ability to demonstrate anatomy through 3D volume-rendered images. The addition of ECG gating minimizes cardiac and bypass graft motion, further improving the sensitivity and specificity of MDCT evaluation of graft patency. These advances have also increased the ability to estimate the extent of intraluminal graft stenosis and occlusion with a noninvasive imaging technique [10]

Chest pain is common after CABG surgery and may be due to recurrent angina secondary to graft occlusion, sternal infection, pleural or pericardial effusion, and less common but potentially lethal complications such as pulmonary embolism or pseudo aneurysm formation. In this setting, MDCT can offer a rapid, convenient, and noninvasive imaging modality to reach the correct underlying diagnosis. In addition, there are several reports on the merits of volume-rendered MDCT images in pre-operative planning for repeating $\mathrm{CABG}$ surgery [3].

One of the most important factors that determine the successful clinical outcome of coronary artery bypass graft surgery is the long-term patency rate of the grafts. For venous coronary artery bypass grafts, an occlusion rate of $12 \%-20 \%$ has been reported within the 1 st year after surgery. After 5 years, the occlusion rate is $4 \%$ per year according to Grondin et al. [11]. On the other hand, the arterial grafts have higher long term patency when compared to venous grafts. For IMA bypass grafts, the occlusion rate is about $10 \%$ after 10 years postimplantation according to Loop [12]. About 40\% of patients who undergo bypass surgery will experience recurrence of symptoms within 6 years, and $25 \%$ of bypass grafts are found to be occluded at 5 years follow-up [13].

Venous grafts are still the most widely used type of grafts due to their availability, accessibility, ease of harvest, resistance to spasm and versatility [14]. In our study, venous grafts were also the most common type of bypass grafts. It represented 40 out of the 63 grafts evaluated, nearly about $63.5 \%$.

It is well known that the main disadvantages of venous grafts are lower patency rates, varicosity, and sclerosis. Furthermore, venous grafts has poor compliance after arterialization, with subsequent atherosclerosis, stenosis and occlusion [15]. Our study showed that $7.5 \%$ of the venous grafts were significantly narrowed and about $65 \%$ of them were occluded. This gives a high rate of complications of about $72.5 \%$. The OM branch was the commonest site for venous graft landing (45\%) followed by the PDA (25\%). The occluded grafts were identified by the characteristic dimple (Nubbin sign) at the site of attachment.

The in-situ arterial grafts are used more common than the free arterial grafts, as the later has more rates of complications [14]. The LIMA is the most widely used in-situ arterial graft and it represented 95.4\% of the in-situ arterial grafts in this study. Due to its arterial nature, it has low incidence of complications and that was evident in this study where $95 \%$ of LIMA grafts were patent without stenosis. On the other hand, the radial artery is the most commonly used free arterial graft due to its easy harvesting. However, it is delicate and highly susceptible to spasm due to its thick muscular wall requiring special care during harvesting [14]

In this study, most of the arterial grafts were in-situ grafts using either right or left internal mammary artery whereas only one free arterial graft was included in this study; the radial artery graft, representing $4.3 \%$ of the arterial grafts.

The LAD is the most common site for landing of the LIMA graft, as it is the most important 
coronary artery branch supplying the left ventricle and due to other anatomical considerations as they are both anteriorly located in the chest [29]. In this study, LAD was the site of landing for $76 \%$ of LIMA grafts. Another landing sites for LAD was the first and second diagonal branches but to a much lesser extent.

The internal mammary artery arises from the subclavian artery, so the scan range has to be extended to cover its origin which increases the radiation dose to the patient compared with venous graft assessment where the upper limit of the scan range can be safely set at aortic arch [12]. In this study, due to lack of operative details at some patients, we set the scan range to include the origin of internal mammary arteries to be sure that they are included in graft assessment just in case they are used as arterial grafts.

In 2001, Ropers et al. [16] reported their experience in the assessment of coronary artery bypass grafts by using retrospectively ECG-gated multidetector row CT angiography using a four detector row machine. In that prospective study, the authors evaluated 65 patients with a total of 182 bypass grafts with conventional coronary angiography as the standard of reference. According to their findings, retrospectively ECG-gated MDCT angiography yielded an excellent diagnostic performance with regard to assessment of occlusion of the graft (sensitivity, 97\%; specificity, 98\%) and a satisfactory diagnostic performance with regard to assessment of stenosis of the bypass graft (sensitivity, $75 \%$; specificity, $92 \%$ ) compared to our study (sensitivity, 100\%; specificity, 95\%). However, a substantial number of non-analyzable coronary bypass grafts was present due to motion artifacts related to the heart, breathing, or movement. The thicker sections used in Willimann et al. [17] study in 2004 and the shorter time for inspiratory breath holding, as well as optimal determination of the reconstruction interval, may have reduced the number of non-analyzable graft segments in their study.

In the study of Ropers et al. [16], out of nine false-positive findings for the detection of bypassrelated high-grade stenosis, six were located at the distal anastomosis of the graft. In this study, all of bypass grafts were interpretable and did not affected by cardiac motion however, two cases showed noninterpretable distal LAD segments beyond graft anastomotic site secondary to cardiac motion due to suboptimal heart rate control.
The arterial grafts are usually accompanied with a lot of surrounding surgical clips that exerts beam hardening and streak artifacts that may make graft lumen assessment difficult. Venous grafts are less susceptible to this problem, mainly due to their larger caliber and the less usage of surgical clips around them. In the study done by Gramer et al. [18], they reported different factors that degrades image quality of coronary artery bypass grafts including stepladder artifacts, poor opacification, small graft segment caliber, surgical clips, motion artifacts and vessel tortuosity. Surgical clips artifacts were present in 5 grafts that interfered with graft lumen assessment. Another study done by Willimann et al. [17] revealed that image quality was reduced because of the presence of metal clip artifacts in five of $62(8 \%)$ coronary artery bypass grafts in five of $20(25 \%)$ patients in their study. The lower frequency of clip artifacts in their study might have also been influenced by different surgical techniques. In this study, surgical clips did not interfere by any means with graft lumen assessment.

Because the data on retrospectively ECG-gated MDCT are acquired with an overlapping helical pitch and continuous X-ray exposure, the applied radiation dose is higher than that in the prospectively ECG-triggered sequential acquisition. However, by reducing the tube current during cardiac cycle phases that are not likely to be used for image interpretation, a dose reduction of up to $48 \%$ is possible according to Jakobs et al. [19]. To reduce radiation dose in this study, we used prospective helical acquisition only exposing 70-80\% of R-R interval in those with heart rate below $60 \mathrm{bpm}$, and $30-80 \%$ of R-R interval in those with heart rate above $60 \mathrm{bpm}$.

In 2008, Hamon et al. [20] made a study with their aim of work to perform a meta-analysis to evaluate the accuracy of 16-and 64-section spiral Computed Tomography (CT) to assess coronary artery bypass grafts. The results of their metaanalysis demonstrate that obstructive bypass graft disease can be detected by using at least a 16section CT with a high diagnostic accuracy with a sensitivity of $98 \%$, a specificity of $97 \%$, with only $92.4 \%$ of scanned grafts being fully assessable. This performance is better than for non-grafted coronary arteries as shown in a meta-analysis by Hamon et al. [20], on a per-vessel basis where the sensitivity was $82 \%$ and specificity was $91 \%$, for the diagnosis of significant coronary artery stenosis. This improvement in performance may be explained by a number of differences between bypass grafts and native coronary arteries. Graft conduits often 
have a larger diameter, are less frequently calcified and are relatively immobile, making CT imaging less challenging. Moreover, grafts are more often occluded than stenosed, which may make the diagnosis of obstruction much easier. Our study agrees with the aforementioned studies as $26(65 \%)$ venous grafts were occluded whereas only 3 (7.5\%) venous grafts showed significant stenosis.

CT images can be reconstructed to yield 3D volume rendered images that gives much anatomical and pathological details in contrast to conventional angiography that only visualize lumen [21]

There are a number of other potential advantages for MDCT over coronary angiography in the assessment of CABG. MDCT is a less invasive, more easy, less time, contrast and complication than coronary angiography. MDCT define accurately the origin of the grafts and offers the surgeon precise information about the position of the existing grafts and the existence of a calcification of the aorta. In patients who require additional aortic valve surgery, an assessment of aortic root, including size and calcification, is also possible [22]

There are a number of limitations of MDCT that need to be considered. There are a substantial number of scanned grafts that are not fully assessable. Reasons for non-assessability are cardiac and respiratory motion artifacts, poor opacification and the presence surgical clips. However, with a 64detector row machine and higher, with increased temporal and spatial resolution and shorter acquisition time, there is an improvement in the rate of fully assessable scanned grafts compared with a 16-section scanner. As regards scan time, up to 40 seconds were needed for a complete assessment of CABG with a four-section scanner. This has been reduced to less than 30 seconds with a 16section scanner and to less than 20 seconds with the 64-section scanner [15]. In our study using 320row CT scanner, the mean scan time was about 5 seconds thus improving image quality.

Even with the use of latest generations CT scanners, there are some limitations that are specific to the evaluation of patients with CABG. First, data acquisition does not provide information about flow characteristics and the functional state of the graft. Second, surgical clips, severe calcifications and extensive atherosclerotic disease, frequently observed in patients with previous CABG, impair the assessment of the native coronary segments as well as few percentage of their grafts. Third, the longer scan range increases the scan time, radiation exposure, and the amount of contrast media needed that further increases the risk of nephrotoxicity [23].

The mean time after the CABG surgery in this study was about 40 months, so we were not be able to assess any of the early complication such as acute and subacute graft occlusion, sternal infection and operative bed collections. All these complications may have been detected if the MDCT study was done shortly after surgery. However, we were mainly dealing with the chronic complication as all patients were referred from outpatient clinics. For studying these types of complications, a study have to be conducted in a hospital and dedicated centers where the CABG operations are done and this may need further future work.

The strength of CTA encompasses a high sensitivity and negative predictive value of 90-99\% for coronary stenosis detection [24].

\section{Conclusion:}

The use of multidetector CT is gaining much acceptance in evaluation of the lumen of coronary artery bypass grafts as it allows better spatial and temporal resolution, shorter scan times, lower radiation dose and contrast material.

\section{References}

1- FRAZIER A.A., QURESHI F., READ K.M., et al.: Coronary artery bypass grafts: Assessment with multidetector CT in the early and late postoperative settings. Radiographics, 25 (4): 881-96, 2005.

2- CAMERON A., DAVIS K.B., GREEN G., et al.: Coronary bypass surgery with internal-thoracic-artery grafts-effects on survival over a 15-year period. The New England Journal of Medicine, 334 (4): 216-9, 1996.

3- KHEDR S.A., HASSAAN M.A. and ALLAM M.H.: Diagnostic value of MDCT angiography in assessment of coronary artery bypass graft. The Egyptian Journal of Radiology and Nuclear Medicine, 44 (2): 183-91, 2013.

4- MALAGUTTI P., NIEMAN K., MEIJBOOM W.B., et al.: Use of 64-slice CT in symptomatic patients after coronary bypass surgery: Evaluation of grafts and coronary arteries. European Heart Journal, 28 (15): 1879-85, 2007.

5- SUN Z., CHOO G.H. and NG K.H.: Coronary CT angiography: Current status and continuing challenges. The British Journal of Radiology, 85 (1013): 495-510, 2012

6- YANG D. and CHOI B.: Imaging of Coronary Revascularization: Stent and CABG. In: Lim T-H, editor. Practical Textbook of Cardiac CT and MRI: Springer Berlin Heidelberg; 103-15, 2015.

7- ABBARA S., ARBAB-ZADEH A., CALLISTER T.Q., et al.: SCCT guidelines for performance of coronary computed tomographic angiography: A report of the Society of Cardiovascular Computed Tomography Guidelines Committee. Journal of cardiovascular computed tomography, 3 (3): 190-204, 2009. 
8- PELBERG R.: Basic Principles in Computed Tomography (CT). Cardiac CT Angiography Manual: Springer London, 19-58, 2015.

9- DEWEY M., ZIMMERMANN E., DEISSENRIEDER F., et al.: Noninvasive Coronary Angiography by 320-Row Computed Tomography With Lower Radiation Exposure and Maintained Diagnostic Accuracy: Comparison of Results With Cardiac Catheterization in a Head-to-Head Pilot Investigation. Circulation, 120 (10): 867-75, 2009.

10- GORANTLA R., MURTHY J.S.N., MURALIDHARAN T.R., et al.: Diagnostic accuracy of 64-slice multidetector computed tomography in evaluation of post-coronary artery bypass grafts in correlation with invasive coronary angiography. Indian Heart Journal, 64 (3): 254-60, 2012.

11- GRONDIN C.M., CAMPEAU L., THORNTON J.C., et al.: Coronary artery bypass grafting with saphenous vein. Circulation, 79: I24-9, 1989.

12- LOOP F.D.: Internal-thoracic-artery grafts. Biologically better coronary arteries. The New England Journal of Medicine, 334 (4): 263-5, 1996.

13- KETONEN M., PAJUNEN P., KOUKKUNEN H., et al.: Long-term prognosis after coronary artery bypass surgery. International Journal of Cardiology, 124 (1): 72-9, 2008.

14- LINDA J. BOGAR S.F. and JAMES T. DICHL: Bypass grafts. In: Halpern EJ, editor. Clinical cardiac CT: Anatomy and function. 2 ed. New York: Thieme, 300-23, 2011.

15- HARSKAMP R.E., LOPES R.D., BAISDEN C.E., et al.: Saphenous vein graft failure after coronary artery bypass surgery: Pathophysiology, management, and future directions. Annals of surgery, 257 (5): 824-33, 2013.

16- ROPERS D., ULZHEIMER S., WENKEL E., et al.: Investigation of aortocoronary artery bypass grafts by multislice spiral computed tomography with electrocardiographic-gated image reconstruction. The American Journal of Cardiology, 88 (7): 792-5, 2001.
17- WILLMANN J.K., WEISHAUPT D., KOBZA R., et al.: Coronary artery bypass grafts: ECG-gated multi-detector row $\mathrm{CT}$ angiography--influence of image reconstruction interval on graft visibility. Radiology, 232 (2): 568-77, 2004.

18- GRAMER B.M., DIEZ MARTINEZ P., CHIN A.S., et al.: 256-slice CT angiographic evaluation of coronary artery bypass grafts: Effect of heart rate, heart rate variability and Z-axis location on image quality. PloS one, 9 (3): e91861, 2014.

19- JAKOBS T.F., BECKER C.R., OHNESORGE B., et al.: Multislice helical CT of the heart with retrospective ECG gating: Reduction of radiation exposure by ECG-controlled tube current modulation. European Radiology, 12 (5): 1081-6, 2002

20- HAMON M., LEPAGE O., MALAGUTTI P., et al.: Diagnostic performance of 16- and 64-section spiral CT for coronary artery bypass graft assessment: Meta-analysis. Radiology, 247 (3): 679-86, 2008.

21- HASSAN W.E.: Role of Multi-detector Row CT Coronary Angiography in the assessment of Coronary Artery Bypass Grafts: Thesis; Cairo University, 2010.

22- FRAZIER A.A., QURESHI F., READ K.M., et al.: Coronary Artery Bypass Grafts: Assessment with Multidetector CT in the Early and Late Post-operative Settings. Radiographics, 25 (4): 881-96, 2005.

23- LEE S.K., JUNG J.I., KO J.M., et al.: Image quality and radiation exposure of coronary $\mathrm{CT}$ angiography in patients after coronary artery bypass graft surgery: Influence of imaging direction with 64-slice dual-source CT. Journal of Cardiovascular Computed Tomography, 8 (2): 124-30, 2014.

24- FEUCHTNER G., KERBER J., BURGHARD P., et al.: The high risk criteria low attenuation plaque lessthan 60 $\mathrm{HU}$ and the napkin ring sign are the most powerful predictors of MACE: Along term follow-up study. Eur. Heart J. Cardiovasc Imaging, 18: 772-9, 2017.

\section{تقييم وصلات الشرايين التاجية بإستخدام الآثعة المقطعية متعددة الكواشف}

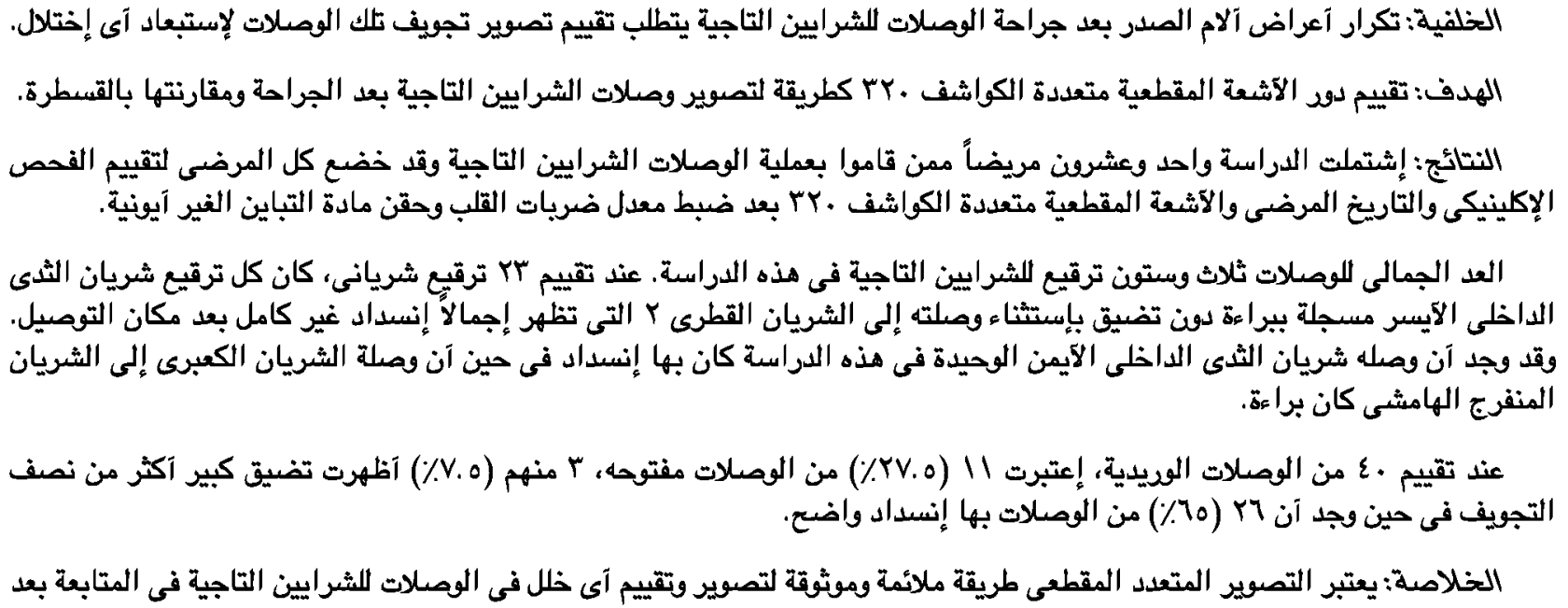

